Pure and Applied Mathematics Quarterly

Volume 8, Number 1

(Special Issue: In honor of

F. Thomas Farrell and Lowell E. Jones, Part 1 of 2)

$107-132,2012$

\title{
Asymptotic Conditions for Smooth Rigidity of Negatively Curved Manifolds
}

\author{
Chris Connell ${ }^{*}$
}

\begin{abstract}
We establish sufficient conditions for a continuous map of nonzero degree between a closed manifold and a negatively curved closed manifold to be homotopic to a smooth covering map, and in particular a diffeomorphism when the degree is one. When the domain manifold is negatively curved, the conditions can be stated in terms of the sphere maps formed by composition of the geodesic projections and the asymptotic boundary map induced by the original map on the universal covers.
\end{abstract}

Keywords: smooth structure, barycenter method, smooth rigidity, negative curvature, quasiconformal, quasi-Möbius.

\section{INTRODUCTION}

A central problem in smooth topology is to determine when a continuous map between two smooth closed manifolds is homotopic to a diffeomorphism. The main purpose of this paper is to give a sufficient criterion of a coarse geometric nature when the target manifold is negatively curved.

When both manifolds are negatively curved the main result roughly states that if the composition of geodesic projections and the asymptotic boundary map of any continuous map $f$ are sufficiently close to being conformal maps between

Received September 27, 2008.

2010 Mathematics Subject Classification: 57R55,53C24,57R45,53C23

*Supported in part by an NSF grant DMS-0608643. 
spheres, then $f$ is homotopic to one which is as nice as possible - a smooth cover. Our approach is to exploit and analyze the barycenter construction developed in this generality by Besson Courtois and Gallot (see e.g. [BCG95, BCG96, BCG99]). As a consequence, the resulting homotopy is fairly explicit.

Let $f: M \rightarrow N$ be a map between closed Riemannian manifolds $(M, g)$ and $\left(N, g_{o}\right)$. When $\pi_{1}(M)$ and $\pi_{1}(N)$ are both Gromov hyperbolic groups, then we can define $\partial_{\infty} \widetilde{M}$ and $\partial_{\infty} \widetilde{N}$ respectively to be the Gromov boundaries of $\pi_{1}(M)$ and $\pi_{1}(N)$ for their word metrics with respect to any finite set of generators. If $f_{*}: \pi_{1}(M) \rightarrow \pi_{1}(N)$ is virtually an isomorphism (i.e. if $f_{*}$ has finite kernel and finite index image), then by [Gro87] the homomorphism $f_{*}: \pi_{1}(M) \rightarrow \pi_{1}(N)$ induces an $f_{*}$-equivariant homeomorphism $\partial f: \partial_{\infty} \widetilde{M} \rightarrow \partial_{\infty} \widetilde{N}$. Since the index $\left[f_{*} \pi_{1}(N), \pi_{1}(M)\right]$ divides $\operatorname{deg}(f)$, it is enough to assume that $f$ has nonzero degree and that $\operatorname{ker} f_{*}$ is finite.

The map $\partial f$ has further structure of an analytic nature once we equip $\partial_{\infty} \widetilde{M}$ and $\partial_{\infty} \widetilde{N}$ with their respective Gromov metrics. Namely, it is bi-Hölder and quasimöbius (see Section 4). When the metrics $g$ and $g_{o}$ are negatively curved, $\partial M$ and $\partial N$ are topological spheres and it is more natural to equip $\partial_{\infty} \widetilde{M}$ and $\partial_{\infty} \widetilde{N}$ with the Gromov boundary metric induced from these metrics instead of the word metrics on $\pi_{1}$. In this case, there is a canonical family of boundary metrics, biLipschitz to the Gromov boundary metrics, for which the boundary action of all isometries is conformal. However, it is important to note that even when $\partial_{\infty} \widetilde{M}$ and $\partial_{\infty} \widetilde{N}$ admit compatible smooth structures, their natural quasiconformal structures usually do not usually belong to the quasiconformal class of the standard conformal structure given by the round metric. This happens already for the rank one symmetric spaces of nonconstant curvature where the Carnot-Carathéodory metrics determine the natural boundary conformal structure.

From now on we will assume that $\left(N, g_{o}\right)$ is negatively curved, and therefore we have projection homeomorphisms $\pi_{x}: S_{x} \widetilde{N} \rightarrow \partial_{\infty} \tilde{N}$ defined by setting $\pi_{x}(v)$ to be the equivalence class of geodesic ray with initial tangent vector $v$. This gives rise to the asymptotic holonomy maps $\pi_{x, y}: S_{x} \widetilde{N} \rightarrow S_{x} \widetilde{N}$ defined by $\pi_{x, y}:=\pi_{y}^{-1} \circ \pi_{x}$. For example, in real hyperbolic space $\mathbb{H}^{n}$, the $\pi_{x, y}$ are simply ordinary Möbius maps. 
For our first main result we will assume that $(M, g)$ and $\left(N, g_{o}\right)$ are both negatively curved and that $f_{*}: \pi_{1}(M) \rightarrow \pi_{1}(N)$ is a virtual isomorphism so that we have the homeomorphism $\partial f$. (These assumptions will be removed in Theorem 1.3.) For all $x \in M$ and $y \in N$, we can intertwine $\partial f$ with projections to obtain natural maps between tangent spheres $\mathcal{Q}_{x, y}: S_{x} M \rightarrow S_{y} N$ defined by $\mathcal{Q}_{x, y}=\pi_{y}^{-1} \circ \partial f \circ \pi_{x}$. (Here and henceforth we will always identify $S_{x} M$ with $S_{x} \widetilde{M}$ and $S_{y} N$ with $S_{y} \widetilde{N}$.) Henceforth we shall call the $\mathcal{Q}_{x, y}$ the asymptotic maps.

The goal of this paper is to obtain a natural condition on the $\mathcal{Q}_{x, y}$ that will guarantee that $f$ is homotopic to a diffeomorphism, or a covering map when $|\operatorname{deg}(f)|>1$. As already indicated, with respect to the Gromov metrics on $\partial_{\infty} \widetilde{M}$ and $\partial_{\infty} \widetilde{N}, \partial f$ is always bi-Holder and quasimöbius (and quasisymmetric). So at first glance it may seem natural to assume that a natural condition would be for $\mathcal{Q}_{x, y}$ to be quasimöbius with some uniform constraint on constants. However, since the corresponding conformal structure on $\partial_{\infty} \widetilde{M}$ and $\partial_{\infty} \widetilde{N}$ will in general be different from the standard one on $S_{x} M$ and $S_{x} N$, we do not know if $\mathcal{Q}_{x, y}$ will even be quasiconformal with respect to the round metrics on $S_{x} M$ and $S_{x} N$. In fact, it follows from the main result of [Yue96a], after making a few standard identifications, that if the $\pi_{x, y}$ are all smooth and uniformly quasiconformal, then $N$ has constant curvature.

Hence we should prefer a condition that only constrains large scale behavior of the $\mathcal{Q}_{x, y}$. This is what we offer:

Theorem 1.1. Let $M$ and $N$ be two oriented closed negatively curved manifolds. There is an $\epsilon>0$ such that for any continuous map $f: M \rightarrow N$ which is a virtual isomorphism on $\pi_{1}$, if the asymptotic maps are $\epsilon$-Möbius, then $f$ is homotopic to a smooth cover of degree $|\operatorname{deg}(f)|$. Moreover, $\epsilon$ depends only on the $C^{1}$ norm of the Margulis function of $M$.

\section{Remarks 1.2.}

- The condition on the $\mathcal{Q}_{x, y}$ need only be checked for a compact set of $x$ and $y$. Alternately, if we are willing to shrink $\epsilon$ by one third, we need only check that a single map $\mathcal{Q}_{x_{o}, y_{o}}$ together with all the asymptotic holonomy maps on $M$ and $N$ separately are $\epsilon$-Möbius. (See Observation 3.3 and the comments after.) 
- The condition of the theorem will be satisfied if we replace the $\epsilon$-Möbius condition with $\lambda$-quasiconformality, for a $\lambda$ sufficiently close to 1 . However this condition governs infinitesimal behavior of the maps making it inherently much stronger.

- The oriented condition can be removed if we are willing to use the appropriate notion of degree of $f$. The proofs are essentially identical.

- While topological rigidity of closed nonpositively curved manifolds hold in general ([FJ93]) starting in dimension five, smooth rigidity for closed manifolds even in the negatively curved category simply fails. Farrell and Jones ([FJ89]) have produced examples of closed manifolds with curvature pinched as close to -1 as desired which are homeomorphic, but not diffeomorphic, to a hyperbolic manifold. (See also [GT87] and [FJ94a].) Moreover, Farrell and Ontaneda ([FO04]) showed that analytic deformation methods for producing smooth maps, provided they behave naturally with respect to taking covers, cannot always recover an existing diffeomorphism between negatively curved manifolds. While the maps we use here, like harmonic maps, share this naturality property, they can still prove useful in the presence of additional assumptions.

A slightly different set of conditions for smooth rigidity along the same lines is provided in Theorem 5.7 in Section 4. In [Con05] we gave volumetric conditions for smooth rigidity, although those require dimension at least 5 . One might also try to interpret the results here as a coarse version of the quasiconformal type boundary conditions for the strong rigidity of negatively and nonpositively curved manifolds we gave in Theorems 1.2-1.5 and 4.3 of [Con03].

We will also generalize the above result to the case when $M$ is arbitrary, and not assumed to be negatively curved. However since we do not have an asymptotic boundary sphere in this case, we are obligated to work with geodesic spheres. We define the geodesic sphere maps $\pi_{x}^{R}: S_{x} M \rightarrow \widetilde{M}$ by $v \mapsto \pi_{x}^{R}(v)=\exp _{x}(R v)$, where the exponential map is taken on the universal cover of $M$. Since $\widetilde{N}$ is a Hadamard space, for each $y \in \widetilde{N}$ we have the map $\operatorname{proj}_{y}: \widetilde{N}-\{y\} \rightarrow S_{y} N$ given by $x \mapsto \frac{\exp _{y}^{-1}(x)}{\left\|\exp _{y}^{-1}(x)\right\|}$. We similarly define for each $x \in M$ and $y \in N$ the map $\mathcal{Q}_{x, y}^{R}: S_{x} M \rightarrow S_{y} N$ by $\mathcal{Q}_{x, y}^{R}=\operatorname{proj}_{y} \circ \tilde{f} \circ \pi_{x}^{R}$ where $\tilde{f}$ is any fixed lift to the universal covers. This is well defined off of the set of points $v \in S_{x} M$ such that $\widetilde{f}\left(\exp _{x}(R v)\right)=y$. In particular, since $f$ is a map of nonzero degree, for almost 
every $R>0$, the $\operatorname{map} \mathcal{Q}_{x, y}^{R}$ is defined on almost all of $S_{x} M$. The following result will be proved in Section 6 .

Theorem 1.3. There is an $\epsilon>0$, depending only on $M$, such that for any continuous map $f: M \rightarrow N$ of nonzero degree between any closed manifold $M$ and any closed negatively curved manifold $N$, if for each pair $(x, y)$, every convergent limit map $\lim _{i \rightarrow \infty} Q_{x, y}^{R_{i}}$ with $R_{i} \rightarrow \infty$ is $\epsilon$ close, off of null sets, to a common $\epsilon$-Möbius map, then $f$ is homotopic to a smooth cover of degree $|\operatorname{deg}(f)|$.

Remark 1.4. The condition in the above theorem can be replaced by the condition that the maps $\bar{Q}_{x, y}$ be $\epsilon$-Möbius where $\bar{Q}_{x, y}$ is a globally well-defined integrated version of the maps $Q_{x, y}^{R}$ given in Section 3 right before Subsection 3.1.

We would like to end the section by mentioning the following surprising result of Farrell and Jones which was our primary inspiration.

Theorem 1.5 (Asymptotic Regularity ([FJ94b])). Let $f: M \rightarrow N$ be a homotopy equivalence between two nonpositively curved smooth manifolds of dimension at least 5. Suppose,

- $\partial_{\infty} \widetilde{M}$ and $\partial_{\infty} \widetilde{N}$ have a natural $C^{1}$ structure (i.e. $\pi_{x, y}$ are $C^{1}$ );

- There is a $C^{1}$ conjugacy $\partial h: \partial_{\infty} \widetilde{M} \cong \partial_{\infty} \widetilde{N}$ of the $\pi_{1}$-actions;

- $\partial h$ extends to a $C^{0}$ semiconjugacy $\widetilde{h}: \widetilde{M} \rightarrow \widetilde{N}$;

- $\chi(M)=0$.

Then $f$ is homotopic to a diffeomorphism.

Since compatible $C^{1}$ structures at infinity are only known to exist for surfaces and quarter pinched negatively curved manifolds, it was the search for some sort of sufficient coarse condition that led to the results in this paper. I would also like to thank Tom Farrell for his encouragement.

\section{The Natural Barycenter Map}

Let $(M, g)$ and $\left(N, g_{o}\right)$ be closed, orientable manifolds and let $f: M \rightarrow N$ be an arbitrary continuous map with degree denoted by $\operatorname{deg}(f)$.

We begin by recalling the construction of the natural maps

$$
F_{s}: M \rightarrow N
$$


due to Besson, Courtois and Gallot in its present form. Here $s$ is a parameter chosen to be larger than the volume growth entropy $h(g)$ of $(\widetilde{M}, g)$ defined by

$$
h(g)=\limsup _{R \rightarrow \infty} \frac{\log \operatorname{Vol} B(x, R)}{R},
$$

where $B(x, R)$ is the geodesic ball of radius $R$ in $\widetilde{M}$. Moreover, since $M$ is compact, the limsup in the definition of $h(g)$ can be replaced by the actual limit, which exists ([Man79]).

Let $\widetilde{f}: \widetilde{M} \rightarrow \widetilde{N}$ denote the lift of $f$ to the universal covers. For each $s>0$ and $x \in \widetilde{M}$ consider the measure $\mu_{x}^{s}$ on $\widetilde{M}$ in the Lebesgue class with density

$$
\frac{d \mu_{x}^{s}}{\operatorname{dvol}_{g}}(z)=e^{-s d(x, z)}
$$

where $d$ is the distance function of $\widetilde{M}$. Recall the definition of the volume growth entropy $h(g)$. For all $s>h(g)$ and all $x \in \widetilde{M}$ the total measure $\left\|\mu_{x}^{s}\right\|$ of $\mu_{x}^{s}$ is finite.

Consider the push-forward measure $\widetilde{f}_{*} \mu_{x}^{s}$ on $\tilde{N}$, and define a measure $\sigma_{x}^{s}$ on $\partial_{\infty} \widetilde{N}$ in the following way. For $z \in \widetilde{N}$, let $\nu_{z}$ be the "visual" or Patterson-Sullivan measures normalized to be probability measures on $\partial_{\infty} \widetilde{N}$ (see [BCG95]), and for $U \subset \partial_{\infty} \widetilde{N}$ measurable define

$$
\sigma_{x}^{s}(U)=\int_{\widetilde{N}} \nu_{z}(U) d\left(\widetilde{f}_{*} \mu_{x}^{s}\right)(z) .
$$

That is, we take $\sigma_{x}^{s}$ to be convolution of the push-forward measure $\widetilde{f}_{*} \mu_{x}^{s}$ with the visual measures $\nu_{z}$. Notice that for all $s, x,\left\|\mu_{x}^{s}\right\|=\left\|\sigma_{x}^{s}\right\|$, so the measure $\sigma_{x}^{s}$ is finite for $s>h(g)$.

For $\theta \in \partial_{\infty} \widetilde{N}$ denote by $B_{\theta}(y)$ the Busemann function of $N$ (normalized so that $B_{\theta}(O)=0$ for some fixed origin $O \in \widetilde{N}$ ) and consider the function on $\widetilde{N}$ defined by

$$
\mathcal{B}_{\sigma_{x}^{s}}(y)=\int_{\partial_{\infty} \widetilde{N}} B_{\theta}(y) d \sigma_{x}^{s}(\theta) .
$$

This is a proper strictly convex function, hence it has a unique minimum [BCG95], which we call the barycenter of the measure $\sigma_{x}^{s}$ and denote by $\operatorname{Bar}\left(\sigma_{x}^{s}\right)$.

This construction can be made more general: Given any finite measure $\lambda$ on $\widetilde{N}$ we can define as in (1) a measure $\sigma_{\lambda}$ as the convolution of $\lambda$ with the family of visual measures. For example the convolution of the Dirac-measure $\delta_{z}$ with 
support $z \in \widetilde{N}$ is the visual measure $\nu_{z}$. Similarly, we can define for every finite measure $m$ of $\partial_{\infty} \widetilde{N}$ the function $\mathcal{B}_{m}$ as in (2). The function $\mathcal{B}_{m}$ is proper and convex if $m$ has no atoms. If this is the case, we define $\operatorname{Bar}(m)$, the barycenter of $m$, to be the unique minimum of $\mathcal{B}_{m}$.

For all $s>h(g)$, the map $\widetilde{F}_{s}: \widetilde{M} \rightarrow \widetilde{N}$ defined by $x \mapsto \operatorname{Bar}\left(\sigma_{x}^{s}\right)$ can be summarized in the following diagram:

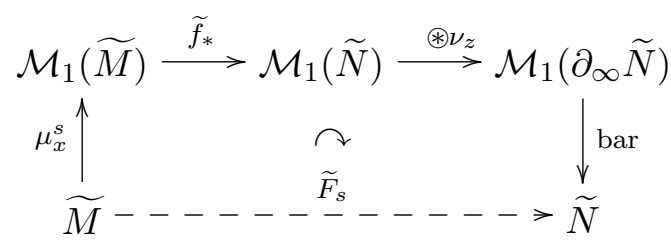

Each stage is equivariant under the intertwined action of $\pi_{1}(M)$ and $f_{*} \pi_{1}(M)<$ $\pi_{1}(N)$. Hence $\widetilde{F}_{s}$ descends to the natural map $F_{s}: M \rightarrow N$.

The following is a collection and restatement of some of the important properties of the natural map due to Besson, Courtois and Gallot [BCG95, BCG98].

Theorem 2.1. Let $(M, g)$ and $\left(N, g_{o}\right)$ be closed orientable $n$-manifolds, let $f$ : $M \rightarrow N$ be a map of nonzero degree and assume that the sectional curvatures of $g_{o}$ are bounded from above by -1 . For each $s>h(g)$ and all $x \in M$,

(1) The natural map $F_{s}$ is at least $C^{1}$.

(2) The map $\widetilde{\Psi}_{s}:[0,1] \times \widetilde{M} \rightarrow \widetilde{N}$ defined by $\widetilde{\Psi}_{s}(t, x)=\operatorname{Bar}\left(t \nu_{\widetilde{f}(x)}+(1-t) \sigma_{x}^{s}\right)$ is equivariant and induces a continuous homotopy between $f$ and $F_{s}$.

(3) If $n \geq 3$, then $\left|\operatorname{Jac}\left(F_{s}\right)(x)\right| \leq\left(\frac{s}{n-1}\right)^{n}$.

Remark 2.2. The appropriate version of the above theorem also holds when $M$ or $N$ are not orientable, assuming that $f$ induces an orientation true homomorphism between the fundamental groups.

\section{Derivatives of the Natural Map}

The barycenter of $\sigma_{\lambda}$ is defined to be the minimum of the $C^{1}$-function $\mathcal{B}_{\sigma_{\lambda}}(\cdot)$. In particular, $\operatorname{Bar}\left(\sigma_{\lambda}\right)=x$ if and only if the gradient of $\mathcal{B}_{\sigma_{\lambda}}$ vanishes at $x$. This gradient can be computed as follows

$$
\nabla_{x} \mathcal{B}_{\sigma_{\lambda}}=\int_{\partial_{\infty} \widetilde{N}} \nabla_{x} B_{\theta} d \sigma_{\lambda}(\theta)=\int_{\widetilde{N}} \int_{\partial_{\infty} \widetilde{N}} \nabla_{x} B_{\theta} d \nu_{z}(\theta) d \lambda(z),
$$


where $\nabla_{x} B_{\theta}$ is the unit vector in $T_{x} \widetilde{N}$ pointing to $\theta \in \partial_{\infty} \widetilde{N}$. Applying this to $\lambda=\mu_{y}^{s}$, we have $\sigma_{\lambda}=\sigma_{y}^{s}$ and the gradient vanishes at $x=\widetilde{F}_{s}(y)$. We denote by $r_{z}$ the function $r_{z}(x)=d(x, z)$. Taking the covariant derivative of the gradient with respect to $y$, i.e. directions $v \in T_{y} \widetilde{M}$, yields

$$
\begin{aligned}
0=D_{v} \nabla_{F_{s}(y)} \mathcal{B}_{\sigma_{y}^{s}}=\int_{\partial_{\infty} \widetilde{N}} & D_{d_{y} F_{s}(v)} \nabla B_{\theta} d \sigma_{y}^{s}(\theta) \\
& \quad-s \int_{\widetilde{M}} \int_{\partial_{\infty} \widetilde{N}} \nabla_{F_{s}(y)} B_{\theta} \otimes d_{y} r_{z}(v) d \nu_{\tilde{f}(z)}(\theta) d \mu_{y}^{s}(z) .
\end{aligned}
$$

Therefore we have,

$$
d_{y} F_{s}=s\left(\int_{\partial_{\infty} \widetilde{N}} D d_{F_{s}(y)} B_{\theta} d \sigma_{y}^{s}\right)^{-1} \int_{\widetilde{M}}\left(\int_{\partial_{\infty} \widetilde{N}} \nabla_{F_{s}(y)} B_{\theta} d \nu_{\tilde{f}(z)}(\theta)\right) \otimes d_{y} r_{z}(y) d \mu_{x}^{s}(z),
$$

where $D d_{F_{s}(y)} B_{\theta}$ is the Hessian of $B_{\theta}$ at the point $F_{s}(y)$. More specifically, it is the self adjoint linear map from $T_{F_{s}(y)} \widetilde{N} \rightarrow T_{F_{s}(y)} \tilde{N}$ such that $D d_{F_{s}(y)} B_{\theta}\left(\nabla_{F_{s}(y)} B_{\theta}\right)=$ 0 and $D d_{F_{s}(y)} B_{\theta}$ restricted to $\left(\nabla_{F_{s}(y)} B_{\theta}\right)^{\perp}$ is the second fundamental form of the horosphere through $F_{s}(y)$ and tangent to $\theta$.

We can rewrite the previous expression more concisely as

$$
d_{y} F_{s}=s \underbrace{\left(\int_{\partial_{\infty} \tilde{N}} D d B_{\theta} d \sigma_{y}^{s}\right)^{-1}}_{A^{-1}} \underbrace{\int_{\widetilde{M}} \int_{\partial_{\infty} \widetilde{N}} \nabla B_{\theta} \otimes d r_{z} d \nu_{\tilde{f}(z)}(\theta) d \mu_{y}^{s}(z)}_{H} .
$$

For any Hadamard space $X$, let $v_{x, z}$ represent the unit vector in $S_{x} X$ tangent to the unique geodesic segment $\gamma_{x, z}$ from $x$ to $z \in X \cup \partial X$. More generally for any manifold $X$, the map $v_{x, .}: X \rightarrow S_{x} X$ makes sense as a Lebesgue measurable function since it is well defined and smooth off of the cut locus of $x$, a subset of Hausdorff codimension at least 1. (Off of the cut locus we choose $\gamma_{x, z}$ to be the unique minimizing geodesic.)

Lemma 3.1. The map $F_{s}: M \rightarrow N$ has a critical point $x \in M$ in the direction $u \in S_{x} M$ if and only if

$$
\int_{\partial_{\infty} \widetilde{N}} v_{y, \theta} d \sigma_{x}^{s}(\theta)=0 \quad \text { and } \quad \int_{\widetilde{M}}\left(\int_{\partial_{\infty} \widetilde{N}} v_{y, \theta} d \nu_{f(z)}(\theta)\right)\left\langle v_{x, z}, u\right\rangle_{x} d \mu_{x}^{s}(z)=0,
$$

for some $y \in M$. (In this case we will necessarily have $y=F_{s}(x)$.)

Proof. Since for $y \in \widetilde{N}$ we have $\nabla_{y} B_{\theta}=-v_{y, \theta}$, the first expression is just the statement that $y=F_{s}(x)$. 
Note that on the orthogonal complement of $\nabla B_{\theta}, D d B_{\theta}$ is the second fundamental form of the horosphere at $\theta$ in $\tilde{N}$. Hence it has one dimensional kernel precisely in the direction of $\nabla_{y} B_{\theta}=-v_{y, \theta}$, and the other eigenvalues are all bounded away from 0 depending only on the upper curvature bound of $N$. Since $d \sigma_{x}$ is supported on all of $\partial_{\infty} \widetilde{N}$, there is no common kernel direction so the tensors $A$ and $A^{-1}$ have no kernel.

Therefore, from the expression of $d_{x} F_{s}$ given above, $F_{s}$ will have a critical direction $u$ if and only if $H(u)=0$. Since $d r_{z}(u)=\left\langle v_{x, z}, u\right\rangle_{x}$, this yields the second expression.

Now suppose for the moment that $(M, g)$ is also negatively curved and that the induced map $f_{*}$ on $\pi_{1}$ has finite kernel. As mentioned in the introduction, $\left[f_{*} \pi_{1}(M): \pi_{1}(N)\right]$ divides $\operatorname{deg}(f)$ and hence $f_{*}$ is a virtual isomorphism since we will assume $\operatorname{deg}(f) \neq 0$. In particular, there is an equivariant homomorphism $\partial f: \partial_{\infty} \widetilde{M} \rightarrow \partial_{\infty} \widetilde{N}$ of the boundary spheres.

Under these assumptions on $M$, the measures $\sigma_{x}^{s}$ limit to the push forward measures $(\partial f)_{*} \nu_{x}$ as $s \rightarrow h(g)$, where $\nu_{x}$ also denotes the Patterson-Sullivan measure on $\partial_{\infty} \widetilde{M}$ (see [BCG96]). The two conditions simplify to

$$
\int_{\partial_{\infty} \tilde{N}} v_{y, \theta} d(\partial f)_{*} \nu_{x}(\theta)=0 \quad \text { and } \quad \int_{\partial_{\infty} \tilde{N}} v_{y, \theta}\left\langle v_{x, \partial f^{-1}(\theta)}, u\right\rangle_{x} d(\partial f)_{*} \nu_{x}(\theta)=0 .
$$

These can be rewritten as

$$
\int_{\partial_{\infty} \widetilde{M}} v_{y, \partial f(\theta)} d \nu_{x}(\theta)=0 \quad \text { and } \quad \int_{\partial_{\infty} \widetilde{M}} v_{y, \partial f(\theta)}\left\langle v_{x, \theta}, u\right\rangle_{x} d \nu_{x}(\theta)=0 .
$$

For any choice of base point $p \in \widetilde{N}$, the general case can be rewritten as

$\int_{\partial_{\infty} \widetilde{N}} v_{y, \theta} d \sigma_{x}^{s}(\theta)=0$ and $\int_{\partial_{\infty} \widetilde{N}} v_{y, \theta}\left(\int_{\widetilde{M}}\left\langle v_{x, z}, u\right\rangle_{x} e^{-h B_{p, \theta}(f(z))} d \mu_{x}^{s}(z)\right) d \nu_{p}(\theta)=0$

Recall the projection maps $\pi_{x}$ defined in the introduction. We will slightly abuse notation. For any measure $\nu$ on $\partial_{\infty} \widetilde{N}$, we also denote the measure $\left(\pi_{x}\right)^{*} \nu$ on $S_{x} N$ by $\nu$ as well. Doing this we can write the previous conditions as,

$$
\int_{S_{y} N} v d \sigma_{x}^{s}(v)=0 \quad \text { and } \quad \int_{S_{y} N} v\left(\int_{\widetilde{M}}\left\langle v_{x, z}, u\right\rangle_{x} e^{-s B_{v}(f(z))} d \mu_{x}^{s}(z)\right) d \nu_{y}(v)=0
$$


In the case $M$ is also negatively curved, the limit map $F:=\lim _{s \rightarrow h} F_{s}$ exists, and the measures $\sigma_{x}^{h}=f_{*} \nu_{x}$, the push forwards of the Patterson-Sullivan measures. We also have radial projection maps $\pi_{x}: S_{x} M \rightarrow \partial_{\infty} \widetilde{M}$. Define the maps $\mathcal{Q}_{x}: S_{x} M \rightarrow S_{F(x)} N$ by $\mathcal{Q}_{x}=\pi_{F(x)}^{-1} \circ \partial f \circ \pi_{x}$. So in this case, we may express the condition for a critical point as

$$
\int_{S_{F(x)} N} v d f_{*} \nu_{x}(v)=0 \text { and } \int_{S_{F(x)} N} v\left\langle\mathcal{Q}_{x}^{-1} v, u\right\rangle_{x} d f_{*} \nu_{x}(v)=0
$$

or finally,

$$
\int_{S_{x} M} \mathcal{Q}_{x}(v) d \nu_{x}(v)=0 \text { and } \int_{S_{x} M} \mathcal{Q}_{x}(v)\langle v, u\rangle_{x} d \nu_{x}(v)=0
$$

The maps $\mathcal{Q}_{x}$ are natural in the sense that if $\widetilde{M}$ and $\widetilde{N}$ are the same rank one symmetric space and $f$ is an isometry, then the $F=f$ and $\mathcal{Q}_{x}$ is simply the linear isometry $d_{x} f$ restricted to the corresponding unit tangent spheres.

Since linear maps commute with (dominated) vector integrals, if $x \in M$ is a critical point for the map $F$ and $L: T_{F(x)} N \rightarrow T_{x} M$ is any linear map, we have

$$
\int_{S_{x} M} L\left(\mathcal{Q}_{x}(v)\right) d \nu_{x}(v)=0 \text { and } \int_{S_{x} M} L\left(\mathcal{Q}_{x}(v)\right)\langle v, u\rangle_{x} d \nu_{x}(v)=0 .
$$

Post composing with arbitrary linear maps also allows us to capture all of the additional obvious symmetries of the situation that we may exploit. For instance, taking the inner product of the original vector valued integrals with a fixed $v_{0} \in T_{F(x)} N$ is equivalent to taking $L$ to be the linear projection to the line $v_{0} \mathbb{R}$ followed by an isometry to $\mathbb{R}$. There are also symmetries of the integrals coming from nonlinear measure preserving transformations, but these are entirely dependent on the measures $\mu_{x}^{s}$ that may arise.

For our immediate purposes, the point of the above observation is to allow us to work on a single space, namely the $(n-1)$-sphere $S_{x} M$. It will be convenient to establish the following convention.

Definition 3.2. We set the map $Q_{x, y}: S_{x} M \rightarrow S_{x} M$ to be the map $Q_{x, y}=$ $L_{x, y} \circ \mathcal{Q}_{x, y}$ where $L_{x, y}: T_{y} N \rightarrow T_{x} M$ is any fixed choice of linear isometry. We similarly define $Q_{x}, Q_{x, y}^{R}$ and $Q_{x}^{R}$. In the latter two cases, we choose the same $L_{x, y}$ for each $R>0$. 
Having distilled the condition in Lemma 3.1, we can begin to more clearly see the nature of the constraint imposed by a critical point. Working in $T_{x} M$, we can approximate integration by the measure $\left(Q_{x}\right)_{*} \nu_{x}$ on $C^{0}\left(S_{x} M\right)$ by the approximating measure $g_{\epsilon} m_{x}$, where $m_{x}$ is the standard Lebesgue measure on $S_{x} M$ and $g_{\epsilon}(v)=\frac{\nu_{x}\left(Q_{x}^{-1}(B(v, \epsilon))\right)}{m_{x}(B(v, \epsilon))}$. Hence writing $q(v):=\langle v, u\rangle_{x}$, both $\int_{S_{x} M} v g_{\epsilon} d m_{x}(v)$ and $\int_{S_{x} M} v q(v) g_{\epsilon}(v) d m_{x}(v)$ should tend to 0 as $\epsilon \rightarrow 0$. This is a somewhat unlikely event, since this last expression says that the average of $\int_{q(v)>0} v|q(v)| g_{\epsilon}(v) d m_{x}(v)$ should almost equal $\int_{q(v)<0} v|q(v)| g_{\epsilon}(v) d m_{x}(v)$. These vectors are respectively the Euclidean centers of mass over the subsets $S^{+}=\left\{v g_{\epsilon}(v)|q(v)|: q(v)>0\right\}$ and $S^{-}=\left\{v g_{\epsilon}(v)|q(v)|: q(v)<0\right\}$. Since $S_{+}$ and $S_{-}$are star convex sets from $0 \in T_{x} M$, we obtain a contradiction if $Q_{x}$ carries the hemisphere determined by $u$ sufficiently close to any hemisphere of $S_{x} M$. We will quantify this situation and weaken such conditions to those of the theorems which do not depend on the intrinsic local nature of the construction.

In the case of a general closed manifold $M$, we will prefer a simpler form of barycenter construction than the one given above, which we will still need and use for the case of a negatively curved $M$.

We shall relabel $\sigma_{x}^{s}=\widetilde{f}_{*} \mu_{x}^{s}$ and then $F_{s}(x)$ will be the unique critical point of the map

$$
y \mapsto \int_{\widetilde{N}} d_{o}(y, z)^{2} d \sigma_{x}^{s}(z)
$$

where $d_{o}$ is the distance function on $\widetilde{N}$. Since $\widetilde{N}$ is a negatively curved Hadamard manifold $d_{o}^{2}$ is smooth everywhere, and the implicitly defined $F_{s}$ is $C^{1}$. We can differentiate $F_{s}$ as before to obtain,

$$
d_{x} F_{s}(u)=-s\left(\int_{\widetilde{N}} d_{o}\left(F_{s}(x), z\right) U_{F_{s}(x)}(z) d \sigma_{x}^{s}(z)\right)^{-1} \circ \int_{\widetilde{M}} \nabla_{F_{s}(x)} d_{o}\left(F_{s}(x), \widetilde{f}(z)\right)
$$

where $U_{F_{s}(x)}(z)$ is the second fundamental form of the geodesic sphere centered at and with radius $d\left(F_{s}(x), z\right)$ evaluated at the point $z$ on the sphere. (For a more detailed account of the derivation of $d_{x} F_{s}$, using this definition of $F_{s}$, see Lemma A.1 of [Sam99].) Since $U_{F_{s}(x)}(z)$ is positive definite in a negatively curved manifold, the entire first integral is strictly positive definite, and so a critical point occurs if and only if the last integral vanishes. 
Using the vector notation $v_{x, z}$ to denote $\nabla_{x} d(x, z)$, we can write this integral condition more simply as,

$$
\int_{\widetilde{M}} v_{F_{s}(x), \widetilde{f}(z)}\left\langle v_{x, z}, u\right\rangle_{x} d \mu_{x}^{s}(z)=0
$$

We can decompose the volume form dvol on $\widetilde{M}$ into the induced spherical volume measures, $\mathrm{Vol}_{r}$ on the geodesic sphere $S(x, r)$, so that for any measurable set $U \subset \widetilde{M}$ we have,

$$
\operatorname{Vol}(U)=\int_{0}^{\infty} \operatorname{Vol}_{r}(U \cap S(x, r)) d r
$$

We therefore obtain a decomposition of the measure $\mu_{x}^{s}$ as

$$
\mu_{x}^{s}(U)=\int_{0}^{\infty} e^{-s r} \operatorname{Vol}_{r}(U \cap S(x, r)) d r .
$$

We now let $\sigma_{x}^{s}$ denote the measure in the class of the Lebesgue measure on $S_{x} M$ such that

$$
d \sigma_{x}^{s}(v)=\int_{0}^{\infty} d \mu_{x}^{s}\left(\exp _{x}(r v)\right) d r
$$

Now let $m_{x}$ denote the standard spherical measure on $S_{x} M$ and $\operatorname{dvol}_{r}(v)$ denote the spherical volume element of radius $r$ in the direction of $v \in S_{x} M$. We can then write for any measurable $U \subset S_{x} M$,

$$
\sigma_{x}^{s}(U)=\int_{0}^{\infty} \int_{U} e^{-s r} \operatorname{dvol}_{r}(v) d m_{x}(v) d r .
$$

Using this we can now write the above condition together with the barycenter condition in terms of the maps $\mathcal{Q}_{x}^{R}$ as,

$$
\begin{array}{r}
\int_{0}^{\infty} \int_{S_{x} M}\langle v, u\rangle_{x} \mathcal{Q}_{x}^{r}(v) e^{-s r} \operatorname{dvol}_{r}(v) d m_{x}(v) d r=0 \\
\int_{0}^{\infty} \int_{S_{x} M} \mathcal{Q}_{x}^{r}(v) e^{-s r} \operatorname{dvol}_{r}(v) d m_{x}(v) d r=0 .
\end{array}
$$

This can be expressed even more simply in terms of the integrated maps with nonspherical range, $J_{x}: S_{x} M \rightarrow T_{x} M$, given by $J_{x}(v)=\int_{0}^{\infty} Q_{x}^{r}(v) e^{-s r} \operatorname{dvol}_{r}(v) d r$.

$$
\int_{S_{x} M}\langle v, u\rangle_{x} J_{x}(v) d m_{x}(v)=0 \text { and } \int_{S_{x} M} J_{x}(v) d m_{x}(v)=0 .
$$


Finally, we may set $\bar{Q}_{x}: S_{x} M \rightarrow S_{x} M$, to be the, possibly discontinuous, map

$$
\bar{Q}_{x}= \begin{cases}\frac{J_{x}(v)}{\left\|J_{x}(v)\right\|} & J_{x}(v) \neq 0 \\ 0 & J_{x}(v)=0 .\end{cases}
$$

(Similarly we may define $J_{x, y}$ and $\bar{Q}_{x, y}$ in the obvious way so that $\bar{Q}_{x}=\bar{Q}_{x, F_{s}(x)}$.) If the measure $\tau_{x}^{s}$ is redefined to be the unique measure satisfying $\tau_{x}^{s}<<m_{x}$ with Radon-Nikodym derivative $\frac{d \tau_{x}^{s}}{d m_{x}}(v)=\left\|J_{x}(v)\right\|$, then the condition for a critical point becomes simply,

$$
\int_{S_{x} M}\langle v, u\rangle_{x} \bar{Q}_{x}(v) d \tau_{x}^{s}(v)=0 \text { and } \int_{S_{x} M} \bar{Q}_{x}(v) d \tau_{x}^{s}(v)=0 .
$$

3.1. Equivariance of the Asymptotic Maps. If we denote by $\Gamma_{M}$ and $\Gamma_{N}$ the image of the natural representations $\pi_{1}(M) \hookrightarrow \operatorname{Isom}(\widetilde{M})$ and $\pi_{1}(N) \hookrightarrow \operatorname{Isom}(\widetilde{N})$, then for each $\gamma \in \Gamma_{M}$ and $x \in \widetilde{M}$ we have $\pi_{\gamma x}=\bar{\gamma} \circ \pi_{x} \circ d \gamma^{-1}$ where $d \gamma^{-1}$ is the derivative map on tangent spheres of the isometry $\gamma^{-1}$ and $\bar{\gamma}$ is the induced map on $\partial_{\infty} \widetilde{M}$. (As we will see shortly this latter map is Möbius with respect to a certain natural class of metrics on $\partial_{\infty} \widetilde{M}$.) The analogous relation holds for $\pi_{\gamma^{\prime} y}$ for all $y \in \widetilde{N}$ and $\gamma^{\prime} \in \Gamma_{N}$.

Similarly, we have $\partial f \circ \bar{\gamma}=\overline{f_{*}(\gamma)} \circ \partial f$. From these observations it follows that for each $x \in \widetilde{M}, y \in \widetilde{N}$ and $\gamma \in \Gamma_{M}$ we have,

$$
\mathcal{Q}_{\gamma x, f_{*}(\gamma) y}=d f_{*}(\gamma) \circ \mathcal{Q}_{x, y} \circ d \gamma^{-1} .
$$

Since the derivatives of isometries are pointwise linear isometries, we see that the $\mathcal{Q}_{x, y}$ are determined by those maps where either $x$ lies within a fundamental domain of $\widetilde{M}$ or $y$ lies in a fundamental domain of $\widetilde{N}$. It is easy to check that a similar equivariance holds in the more general case for the $\mathcal{Q}_{x, y}^{R}$. Since the range of the map $F$ and the maps $F_{s}$ on a fundamental domain are compact, we have

Observation 3.3. The conditions on the $\mathcal{Q}_{x, y}$ and $\mathcal{Q}_{x, y}^{R}$ in Theorems 1.1-1.3 need only be checked for a compact set of $x$ and $y$.

As an immediate consequence of the definitions, for any $x, x^{\prime} \in \widetilde{M}$ and $y, y^{\prime} \in \widetilde{N}$ we have

$$
\mathcal{Q}_{x^{\prime}, y^{\prime}}=\pi_{y, y^{\prime}} \circ \mathcal{Q}_{x, y} \circ \pi_{x^{\prime}, x} .
$$

Hence the maps $\mathcal{Q}_{x, y}$ are entirely determined by the $\mathcal{Q}_{x, y}$ for $x$ in a fundamental domain $\mathcal{F}_{M}$ for $\Gamma_{M}$ and $y$ in a fundamental domain $\mathcal{F}_{N}$ for $\Gamma_{N}$ together with the 
maps $\pi_{x, \gamma x}$ (respectively $\pi_{y, \gamma^{\prime} y}$ ) where $x \in \mathcal{F}_{M}$ (resp. $y \in \mathcal{F}_{N}$ ). Similarly, the $\mathcal{Q}_{x, y}$ are determined by any single map $\mathcal{Q}_{x_{o}, y_{o}}$ together with all of the asymptotic holonomy maps $\pi_{x, x^{\prime}}$ and $\pi_{y, y^{\prime}}$ for $x, x^{\prime} \in \widetilde{M}$ and $y, y^{\prime} \in \widetilde{N}$.

Despite the fact that $\gamma$ is an isometry and $\bar{\gamma}$ acts by Möbius transformations with respect to the natural choice of metric on $\partial_{\infty} \widetilde{M}$, one should bear in mind that the maps $\pi_{x, \gamma x}$ are rarely smooth, let alone Möbius with respect to the standard conformal structure. More specifically, they are in general only $\frac{1}{\kappa}$-Hölder when the metric is $\frac{1}{k^{2}}$-pinched ([AS85]). However, they are always smooth for surfaces and $C^{1}$ in the quarter pinched case.

\section{Quasimöbius AND $\epsilon$-MöBIUs MAPS}

In this section we mainly recall the some aspects of quasiconformal maps and the asymptotic geometry of negatively curved manifolds.

We begin more generally with a general $\operatorname{CAT}(-1)$ space $X$, such as $\widetilde{M}$. The Gromov product is the quantity

$$
(x \cdot y)_{p}:=\frac{1}{2}(d(p, x)+d(p, y)-d(x, y)), \forall p, x, y \in X .
$$

In this setting, the product extends continuously to the compactification $\bar{X}=$ $X \cup \partial_{\infty} X$. The

Now we define the visual metric at $p \in X$ to be

$$
d_{p}(x, y)=e^{-(x \cdot y)_{p}} .
$$

Bourdon ([Bou95]) showed that this is always an honest metric.

Recall that a map $f:(Z, d) \rightarrow\left(Z^{\prime}, d^{\prime}\right)$ is quasiconformal if there exists $K \in$ $[1, \infty)$ such that for each $x \in Z$,

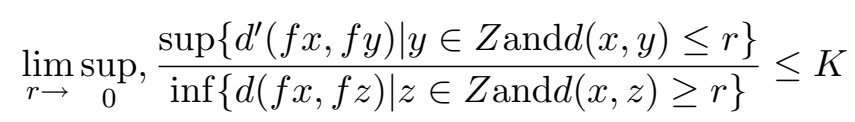

We Define the cross-ratio on $\partial_{\infty} X$ via $d_{p}$, i.e.

$$
[x, y, z, w]:=\frac{d_{p}(x, z) d_{p}(y, w)}{d_{p}(x, w) d_{p}(y, z)} .
$$


This expression only makes sense for pairwise distinct points $x, y, z, w$ in $\partial_{\infty} X$. It can also be defined in the larger domain $\bar{X}$, and it is independent of the choice of $p$ (see [Pau96]). A map is called Möbius if it preserves the cross-ratio.

If one puts the metric $d_{p}$ on the ideal boundary of the CAT $(-1)$ space $X$, then the homomorphism of $\partial X$ induced by $g \in \operatorname{Isom}(X)$ is Möbius, bi-Lipschitz and conformal ([Bou95]).

Definition 4.1. Given two metric spaces $(X, d)$ and $\left(Y, d^{\prime}\right)$, a homeomorphism $f: X \rightarrow Y$ is called $\eta$-quasisymmetric with respect to a homeomorphism $\eta$ : $[0, \infty) \rightarrow[0, \infty)$ if

$$
\frac{d^{\prime}(f(x), f(y))}{d^{\prime}(f(x), f(z))} \leq \eta\left(\frac{d(x, y)}{d(x, z)}\right)
$$

for all triples $x, y, z \in X$.

Definition 4.2. Given two metric spaces $(X, d)$ and $\left(Y, d^{\prime}\right)$, a homeomorphism $f: X \rightarrow Y$ is called $\eta$-quasimöbius with respect to a homeomorphism $\eta:[0, \infty) \rightarrow$ $[0, \infty)$ if

$$
[f(x), f(y), f(z), f(w)] \leq \eta([x, y, z, w]),
$$

for all quadruples $x, y, z, w \in X$.

In the above two definitions two metrics $d$ and $d^{\prime}$ on $X$ are $\eta$-quasisymmetric (resp. $\eta$-quasimöbius) if the identity map has corresponding property. Similarly, we say that a map or metric is quasisymmetric (resp. quasimöbius) if it is $\eta$ quasisymmetric (resp. $\eta$-quasimöbius) for some $\eta$.

Definition 4.3. A space $X$ is said to be homogeneously dense if there is a number $\lambda$ such that $0<\lambda<1$ and for each pair of points $a, b \in X$ there is a point $x \in X$ satisfying the condition $d(x, a)<\lambda d(a, b)$ and $d(x, b)<\lambda d(a, b)$.

Lemma 4.1. The space $\left(\partial_{\infty} X, d_{p}\right)$ is homogeneously dense for each $p$.

Proof. For each pair $a, b \in \partial_{\infty} X$ let $q$ be the point on the geodesic $[a, b]$ closest to $p$. Take $x$ to be the endpoint of the geodesic ray from $p$ to $q$. Let $r$ be the point on $[a, x]$ furthest from $[a, q] \cup[q, x]$. Since $\angle_{q}(a, x)=\frac{\pi}{2}$, there is a universal constant $C$ such that $d(q, r)<C$ so $|d(p, r)-d(p, q)| \leq C$. By Gromov $\delta$-hyperbolicity of $X,\left|d(p,[a, b])-(a, b)_{p}\right| \leq 2 \delta$. Consequently we can take $\lambda=e^{-C-8 \delta}$. 


\subsection{Quasimöbius Maps.}

Definition 4.4. For any $k>0$ the term k-quasimöbius (respectively $k$-quasisymmetric) will mean $\eta$-quasimöbius (respectively $\eta$-quasisymmetric) for the homeomorphism $\eta(t)=k * t^{\frac{1}{k}}$.

The following result shows that the more restricted notion of $k$-quasisymmetric or Möbius is equivalent to the more general $\eta$-version.

Proposition 4.2 (Tukia and Vaisala [Väi71]). For any homogeneously dense metric space for any $\eta$-quasisymmetric ( $\eta$-quasimöbius) map $f$ there is a $k$ such that $f$ is k-quasisymmetric (k-quasimöbius).

The property of being quasimöbius is the natural analogue on $S^{n}$ of the property of being quasisymmetric $\mathrm{n} \mathbb{R}^{n}$. Indeed, if $\mathrm{St}_{a}: S^{n} \backslash\{a\} \rightarrow \mathbb{R}^{n}$ is the (conformal) stereographic projection from the point $a \in S^{n}$, then $f: S^{n} \rightarrow S^{n}$ is quasimöbius with respect to the standard round metric if and only if $\mathrm{St}_{b} \circ f \circ \mathrm{St}_{a}^{-1}$ is a quasisymmetric map for all $a, b \in S^{n}$.

In fact, we can make this analogy precise for an arbitrary Gromov hyperbolic space $X$. For $x \in X$ and any fixed $a \in \partial_{\infty} \widetilde{X}$, following [Min07] we define the stereographic projection of $d_{x}$ with respect to $a$ by

$$
d_{x \mid a}(y, z):=\frac{d_{x}(y, z)}{d_{x}(y, a) d_{x}(z, a)}, y, z \in \partial_{\infty} \tilde{X} \backslash\{a\} .
$$

When $d_{x}$ is replaced by the round metric, this formula agrees with the formula from elementary Euclidean geometry for the classical stereographic projection. The following establishes the main property of the stereographic projection.

Proposition 4.3 (Theorem 16 of [Min07]). The function $d_{x \mid a}$ is a metric on $\partial X \backslash\{b\}$ conformally equivalent to $d_{x}$. The metrics $d_{x}$ and $d_{x \mid a}$ induce the same (usual) topology on $\partial X \backslash\{b\}$.

In particular quasimöbius maps with respect to $d_{x}$ are quasisymmetric with respect to $d_{x \mid a}$ for any $a \in \partial_{\infty} X$. We now introduce some related definitions for later use.

Definition 4.5. We say that a map $g: S^{n} \rightarrow S^{n}$ is $\theta$-antipodal if $\angle(g(v),-g(-v)) \leq$ $\theta$ for all $v \in S^{n}$. 
Definition 4.6. We say that a (not necessarily continuous) map $f: S^{n} \rightarrow S^{n}$ is $\epsilon$-coarsely Möbius, or simply $\epsilon$-Möbius, if it is pointwise $\epsilon$-close to a Möbius map, with respect to the standard round metric on $S^{n}$.

Definition 4.7. Given any map $f: X \rightarrow Y$ between two metric spaces $(X, d)$ and $\left(Y, d^{\prime}\right)$, we say that $f$ is $\epsilon$-spherical if the image of every $d$-ball is contained in the $\epsilon$-neighborhood of some $d^{\prime}$-ball. We say two metrics $d, d^{\prime}$ on $X$ are $\epsilon$-spherical if both the identity map from $(X, d)$ to $\left(X, d^{\prime}\right)$ and from $\left(X, d^{\prime}\right)$ to $(X, d)$ are $\epsilon$-spherical.

4.2. Sullivan's shadow lemma. Fix a point $p \in X$. For any $x \in X$ and

$R>0$ consider the shadow of the ball $B(x, R)$ from $p$ to $\partial_{\infty} X$ defined by

$$
\mathcal{O}_{p}(x, R)=\left\{a \in \partial_{\infty} X: \gamma_{p, a} \cap B(x, R) \neq \emptyset\right\},
$$

where $\gamma_{p, a}$ is the geodesic from $p$ to $a$.

The following result is due to Sullivan in the constant curvature case:

Lemma 4.4 (Sullivan's shadow lemma ([Sul84],[Rob03],[Yue96b])). For all $R>$ 0 , there exists a constant $C=C(R)>1$ such that for all $p \in X$ and all $\gamma \in \Gamma$ we have

$$
C^{-1} e^{-h d(p, \gamma p)} \leq \nu_{p}\left(\mathcal{O}_{p}(\gamma p, R)\right) \leq C^{-1} e^{-h d(p, \gamma p)}
$$

If $a \in \partial_{\infty} X$ is the endpoint of the geodesic from $p$ passing through $\gamma p$ then there is a $C>1$ such that $B_{d_{p}}\left(a, C^{-1} e^{-d(p, \gamma p)}\right) \subset \mathcal{O}_{p}(\gamma p, 1) \subset B_{d_{p}}\left(a, C e^{-d(p, \gamma p)}\right)$. Hence the Sullivan Lemma can be restated as saying

Corollary 4.5. There exists a constant $C>1$ such that for all $p \in X$ we have

$$
\frac{1}{C} r^{h} \leq \nu_{p}\left(B_{d_{p}}(a, r)\right) \leq C^{-1} r^{h}
$$

for all $a \in X$ and $r \leq 1$

As a consequence, for each $p \in X, \nu_{p}$ is in the class of the $h$-dimensional Hausdorff measure $\mathcal{H}_{p}$ for the metric $d_{p}$. Using the fact that $\Gamma$ acts $\nu_{p}$-ergodically on $\partial_{\infty} X$, it was shown in [Ham89] that for the right choice of metric bilipschitz to $d_{p}$, the corresponding $h$-dimensional Hausdorff measure is a constant multiple of $\nu_{p}$. 


\section{Rigidity of the Asymptotic Maps}

5.1. Properties of the $\mathcal{Q}_{x}$ maps. While not necessary for the proof of the main theorems, the next two lemmas give some control on the asymptotic map at the barycenter point of a Patterson-Sullivan measure, and therefore may be of independent interest.

Lemma 5.1. Choose any $1 \geq m \geq \frac{1}{2}$ and let $B \subset S_{x} M$ be any set with $\nu_{x}\left(\pi_{x}(B)\right) \geq m\left\|\nu_{x}\right\|$. The image under $\mathcal{Q}_{x}$ of $B$ cannot be contained in any spherical ball of radius less than $\theta=\cos ^{-1}\left(\frac{1-m}{m}\right)$.

Proof. Suppose not, then we claim that the magnitude of the vector

$$
w=\int_{\mathcal{Q}_{x}(B)} v d \pi_{F(x)}^{*}(\partial f)_{*} \nu_{x}
$$

would be larger than $m \cos (\theta)$. To see this, first suppose that we allowed $\pi_{F(x)}^{*}(\partial f)_{*} \nu_{x}$ to be any measure at all. Note that since we assumed $\theta \leq \frac{\pi}{2}$, any measure with minimal $\|w\|$ must have the property that the intersection of its support with $\mathcal{Q}_{x}(B)$ lies on an angular sphere $S(u, \theta)$ of radius $\theta$ centered at some point $u \in S_{F(x)} N$ containing $\mathcal{Q}_{x}(B)$, and that it takes equal values on opposing subsets of $S(u, \theta)$. In particular we must have $\operatorname{diam}\left(\mathcal{Q}_{x}(B)\right)=2 \theta$. Hence, in any minimal case, we have

$$
w=\frac{1}{2} \pi_{F(x)}^{*}(\partial f)_{*} \nu_{x}\left(\mathcal{Q}_{x}(B)\right) 2 \cos (\angle(u, w)) u=m \cos (\theta)\left\|\nu_{x}\right\| u .
$$

On the other hand, in order for $F(x)$ to be the barycenter point, we must have the vector average over a subset of the hemisphere defined by $\{u: \angle(u, w)<0\}$ canceling $w$. However, even if the remaining mass were concentrated at $-\frac{w}{\|w\|}$, it would not be sufficient. For if $(1-m)\left\|\nu_{x}\right\|=\|w\| \geq m \cos (\theta)\left\|\nu_{x}\right\|$, then this contradicts $\theta<\cos ^{-1}\left(\frac{1-m}{m}\right)$.

Lemma 5.2. The maps $\mathcal{Q}_{x}: S_{x} M \rightarrow S_{F(x)} N$ are always $\theta$-antipodal for some $\theta$ depending only on the constants in the Shadow Lemma.

Proof. Suppose $d_{F(x)}\left(\partial f\left(\pi_{x}(v)\right), \partial f\left(\pi_{x}(-v)\right)\right)<t$ for some value $t<<1$. Since $d_{p}\left(\pi_{x}(v), \pi_{x}(-v)\right)=1$, every $d_{x}$ ball containing both $v$ and $-v$ has radius at least $\frac{1}{2}$. Since $\partial f$ is $\lambda$-quasimöbius with respect to $\pi_{x}^{*} d_{x}$ and $\pi_{F(x)}^{*} d_{F(x)}$, at least one of these balls is sent into a $\pi_{F(x)}^{*} d_{F(x)}$-ball of radius at most $\lambda t$ centered at some point $w \in S_{F(x)} N$. By the Shadow Lemma 4.4 for some $K>1$ a ball containing 
$m=\frac{\sqrt{2}}{1+\sqrt{2}}$ fraction of the total mass of $\nu_{x}$ is sent into the $\pi_{F(x)}^{*} d_{F(x)}$-ball of radius $(1+\epsilon) K t$. However, for $t$ sufficiently small this will lie in a spherical ball of radius $\frac{\pi}{4}$. This contradicts the previous lemma.

Hence $d_{F(x)}\left(\partial f\left(\pi_{x}(v)\right), \partial f\left(\pi_{x}(-v)\right)\right)$ is larger than some uniform constant, and so $\angle_{F(x)}\left(\mathcal{Q}_{x}(v), \mathcal{Q}_{x}(-v)\right)>\pi-\theta$ for some uniform $\theta$ depending only on the quasimöbius constants and the diameter of $N$.

For $x \in M$, let $c(x)$ denote the Margulis function at the point $x \in M$. If $B(\widetilde{x}, R)$ represents the ball of radius $R$ in $\widetilde{M}$ for any lift $\widetilde{x}$ of $x$, then we may define $c(x)$ by

$$
c(x):=\lim _{R \rightarrow \infty} \frac{\operatorname{Vol} B(x, R)}{e^{h R}}
$$

where the limit exists by [Mar70]. By [Yue91] this function is smooth and can be expressed as

$$
\frac{c(x)}{c(p)}=\left\|\nu_{x}\right\|=\int_{\partial_{\infty} \widetilde{M}} e^{h B(x, p, \xi)} d \nu_{p}(\xi)
$$

Hence we can express the gradient as

$$
\frac{\nabla c(x)}{c(p)}=\int_{S_{x} M} v d \nu_{x}(v)
$$

In other words $\nabla c(x)=0$ if and only if the barycenter of $\operatorname{bar}\left(\nu_{x}\right)=x$.

Example 5.3. Suppose that for some $x \in M$ the transformation $Q_{x}$ is Möbius. For any fixed $u \in S_{x} M, Q_{x}$ sends some level set of the function $v \mapsto\langle v, u\rangle$ to an equator sphere. If the measure $\nu_{x}$ were supported on such a level set, then we would immediately have that $u \in \operatorname{ker} d_{x} F$. This follows from the fact that in this case the two integral conditions are just scalar multiples of each other, so they are zero simultaneously.

This degenerate situation cannot occur however, since $\nu_{x}$ cannot be supported on anything less than the whole sphere. Nevertheless, how uniformly $\nu_{x}$ is supported on $S_{x} M$ is controlled by the constants arising in the Shadow Lemma 4.4 together with the properties of the metric $d_{x}$. This also affects how far $Q_{x}$ can vary from being Möbius. 


\subsection{Proof of Theorem 1.1.}

Lemma 5.4. If $Q_{x}$ is Möbius then $x$ is not a critical point for $F$.

Proof. Suppose $u \in \operatorname{ker} d_{x} F$, and let $\left\{S_{t}\right\}$ denote the partition of $S_{x} M$ by level sets of the function $t(v)=\langle v, u\rangle$. Since $Q_{x}$ is Möbius, there is a unique sphere $S_{t_{0}}$ for some $t_{o} \in(-1,1)$ which is sent to an equator sphere by $Q_{x}$. Let $\left\{v_{+}, v_{-}\right\}$ be the two (antipodal) unit vectors orthogonal to $Q_{x}\left(S_{t_{0}}\right)$ and let $H_{+}$and $H_{-}$be the hemispheres corresponding to $v_{+}$and $v_{-}$respectively. (Here we assume we have chosen $v_{+}$such that $Q_{x}(u) \in H_{+}$.)

Recalling our aforementioned notational convenience, we will allow $\nu_{x}$ to also denote the Patterson-Sullivan measure on $\partial_{\infty} \widetilde{M}$ projected to $S_{x} M$, namely $\left(\pi_{x}^{-1}\right)_{*} \nu_{x}$. After splitting the integral conditions (3) on $F$ into hemispheres and taking the inner product with $v_{+}=-v_{-}$, they imply

$$
\int_{Q_{x}^{-1}\left(H_{+}\right)}\left\langle Q_{x}(v), v_{+}\right\rangle d \nu_{x}(v)=\int_{Q_{x}^{-1}\left(H_{-}\right)}\left\langle Q_{x}(v), v_{-}\right\rangle d \nu_{x}(v)
$$

and

$$
\int_{Q_{x}^{-1}\left(H_{+}\right)}\langle v, u\rangle\left\langle Q_{x}(v), v_{+}\right\rangle d \nu_{x}(v)=\int_{Q_{x}^{-1}\left(H_{-}\right)}\langle v, u\rangle\left\langle Q_{x}(v), v_{-}\right\rangle d \nu_{x}(v) .
$$

However, the function $\langle v, u\rangle$ is monotone on the partition $\left\{S_{t}\right\}$ and $\cup_{t>t_{0}} S_{t} \subset$ $Q_{x}^{-1}\left(H_{+}\right)$while $\cup_{t<t_{0}} S_{t} \subset Q_{x}^{-1}\left(H_{-}\right)$. Hence we may compute,

$$
\begin{aligned}
\int_{Q_{x}^{-1}\left(H_{+}\right)}\langle v, u\rangle\left\langle Q_{x}(v), v_{+}\right\rangle d \nu_{x}(v) & >\left(\inf _{v \in Q_{x}^{-1}\left(H_{+}\right)}\langle v, u\rangle\right) \int_{Q_{x}^{-1}\left(H_{+}\right)}\left\langle Q_{x}(v), v_{+}\right\rangle d \nu_{x}(v) \\
& =\left(\inf _{v \in Q_{x}^{-1}\left(H_{+}\right)}\langle v, u\rangle\right) \int_{Q_{x}^{-1}\left(H_{-}\right)}\left\langle Q_{x}(v), v_{-}\right\rangle d \nu_{x}(v) \\
& =\left(\sup _{v \in Q_{x}^{-1}\left(H_{-}\right)}\langle v, u\rangle\right) \int_{Q_{x}^{-1}\left(H_{-}\right)}\left\langle Q_{x}(v), v_{-}\right\rangle d \nu_{x}(v) \\
& >\int_{Q_{x}^{-1}\left(H_{-}\right)}\langle v, u\rangle\left\langle Q_{x}(v), v_{-}\right\rangle d \nu_{x}(v) .
\end{aligned}
$$

The strictness of the inequalities follow from the fact that the measure $\nu_{x}$ is supported on the entire sphere $S_{x} M$. 
Lemma 5.5. In the proof of the previous lemma, the value of $t_{0}$ tends to 0 uniformly as $\left\|\nabla_{x} c(x)\right\|$ tends to 0 .

Proof. If $\nabla c(x)=c(p) \int_{S_{x} M} v d \nu_{x}(v)$ is 0 , then since $Q_{x}$ sends spheres to spheres, it must take every hemisphere to every hemisphere in order for the barycenter condition to be fulfilled. In particular $Q_{x}$ is an isometry since we are already assuming it to be Möbius. Moreover, the size of the smallest neighborhood carried into a hemisphere by $Q_{x}$ is continuous in $\left\|\nabla_{x} c(x)\right\|$, uniformly in $x$. The lemma immediately follows from this.

The natural normalization for the gradient of the Margulis function is

$$
\frac{\nabla_{x} c(x)}{c(x)}=\frac{\nabla_{x} c(x)}{c(p)\left\|\nu_{x}\right\|}=\frac{\nabla_{x}\left\|\nu_{x}\right\|}{\left\|\nu_{x}\right\|}=\frac{1}{\left\|\nu_{x}\right\|} \int_{\partial_{\infty} \widetilde{M}} v d \nu_{x} .
$$

In other words, this is just the $\nu_{x}$ vector average.

Lemma 5.6. There is a continuous monotone function $\theta:[0,1) \rightarrow\left(0, \frac{\pi}{2}\right]$ with $\theta(0)=\frac{\pi}{2}$ such that for all $\delta<1$ if $\frac{\left\|\nabla_{x} c(x)\right\|}{c(x)}<\delta$ and $Q_{x}$ is $\epsilon$-Möbius, then $Q_{x}$ maps every ball of angular radius $\theta(\delta)-\epsilon$ into an open hemisphere.

Proof. From the previous lemma we just need observe that $\frac{\left\|\nabla_{x} c(x)\right\|}{c(x)}<1$ and so if $\epsilon=0$ then $Q_{x}$ always carries every $\theta$-ball into a hemisphere by compactness. On the other hand, from the definition, if $Q_{x}$ is $\epsilon$-Möbius then $Q_{x}$ will still carry every $\theta-\epsilon$ ball into a hemisphere. The fact that $\theta(0)=\frac{\pi}{2}$ follows from Lemma 5.5 .

Proof of Theorem 1.1. Let $\mathcal{M}_{x}$ be a Möbius map that is closest to $Q_{x}$ in the uniform Hausdorff metric.

Set $\delta=\sup _{x \in M} \frac{\left\|\nabla_{x} c(x)\right\|}{c(x)}$. By Lemma 5.6 as $\delta$ tends to 0 , the map $Q_{x}$ becomes $\epsilon$-Hausdorff close to an isometry of the sphere, and uniformly in $\delta$.

Consequently, there is a function $\epsilon(\delta)$ increasing to $\epsilon(0)$ such that whenever $\epsilon<\epsilon(\delta)$, the difference

$$
\int_{Q_{x}^{-1}\left(H_{+}\right)}\langle v, u\rangle\left\langle Q_{x}(v), v_{+}\right\rangle d \nu_{x}(v)-\int_{Q_{x}^{-1}\left(H_{-}\right)}\langle v, u\rangle\left\langle Q_{x}(v), v_{+}\right\rangle d \nu_{x}(v)
$$

from the inequality in the proof of Lemma 5.4 will be positive which produces the desired contradiction. 
We can also give alternate hypotheses in Theorem 1.1 which exploit the fact that $\partial f$ is always $\lambda$-quasimöbius with respect to the $d_{x}$ metrics.

Theorem 5.7. There exist positive numbers $\epsilon>0$ and $\lambda>1$ with the property that if $f: M \rightarrow N$ is any continuous map of nonzero degree between two closed negatively curved manifolds such that such that

(1) $\partial f: \partial_{\infty} \widetilde{M} \rightarrow \partial_{\infty} \widetilde{N}$ is $\lambda$-quasimöbius, and

(2) for each $p \in M$ and $p \in N$, the metrics $\pi_{p}^{*} d_{p}$ and $\angle_{p}$ are $\epsilon$-spherical,

then $f$ is homotopic to a smooth cover $F: M \rightarrow N$ of degree $\operatorname{deg}(f)$.

Here the bounds on $\lambda$ and $\epsilon$ can be given as a single bound on what $(\lambda-1)+\epsilon$ can be. Note also that the hypotheses do not automatically imply that $Q_{x}$ is $\delta$-Möbius for any $\delta>0$ on the scale of $(\lambda-1)+\epsilon$ since both the $\epsilon$-spherical and $\lambda$-qausimöbius conditions only apply to spheres individually and not to the entire map.

Proof. Once again suppose $u \in \operatorname{ker} d_{x} F$, and let $\left\{S_{t}\right\}$ denote the partition of $S_{x} M$ by level sets of the function $t(v)=\langle v, u\rangle$.

The maps $\pi_{y}^{-1} \pi_{x}$ are conformal with respect to $\pi_{x}^{*} d_{x}$ and $\pi_{y}^{*} d_{y}$ for pairs of points $x, y \in M$ or $x, y \in N$. Hence the conditions imply that there is a $\delta$ that depends on $\epsilon$ and $\lambda$ such that the the spheres $S_{t}$ are sent by $Q_{x}$ into $\delta$-neighborhoods of round spheres. On the other hand since $Q_{x}$ is a homeomorphism, one of these is in the $\delta$-neighborhood of a single hemisphere.

The rest of the proof then mimics that of the proof of Theorem 1.1.

\section{The General Case for $M$}

In this section we prove Theorem 1.3. We recall that we are assuming that $M$ is an arbitrary smooth closed manifold and that we had a decomposition of the measure $\mu_{x}^{s}$ from Section 3 as

$$
\mu_{x}^{s}(U)=\int_{0}^{\infty} e^{-s r} \operatorname{Vol}_{r}(U \cap S(x, r)) d r .
$$

With this notation we can now state, 
Lemma 6.1. for any compact subset $U \subset \widetilde{M}$ and any $\epsilon>0$, we can choose an s sufficiently close to $h(g)$ such that $\frac{\mu_{x}^{s}(U)}{\left\|\mu_{x}^{s}\right\|}<\epsilon$.

Proof. Since $U \cup S(x, R)$ for some $R>0$, we have $\mu_{x}^{s}(U) \leq \int_{0}^{R} e^{-s r} \operatorname{Vol}_{r}(S(x, r)) d r$. So it remains to show that $\left\|\mu_{x}^{s}\right\|=\int_{0}^{\infty} e^{-s r} \operatorname{Vol}_{r}(S(x, r)) d r$ tends to $\infty$ as $s \rightarrow h(g)$.

Note that

$$
\begin{aligned}
\int_{0}^{\infty} e^{-s r} \operatorname{Vol}_{r}(S(x, r)) d r & =\int_{0}^{\infty} e^{-s r} \frac{d}{d r} \operatorname{Vol}(B(x, r)) d r \\
& =\int_{0}^{\infty} \frac{d}{d r}\left(e^{-s r} \operatorname{Vol}(B(x, r))\right) d r \\
& -\int_{0}^{\infty} \frac{d}{d r}\left(e^{-s r}\right) \operatorname{Vol}(B(x, r)) d r \\
& =\left.e^{-s r} \operatorname{Vol}(B(x, r))\right|_{0} ^{\infty}+s \int_{0}^{\infty} e^{-s r} \operatorname{Vol}(B(x, r)) d r \\
& =s \int_{0}^{\infty} e^{-s r} \operatorname{Vol}(B(x, r)) d r .
\end{aligned}
$$

So it is sufficient to show that $\int_{0}^{\infty} e^{-h r} \operatorname{Vol}(B(x, r)) d r$ is unbounded.

Let $\Gamma$ be the image of the covering representation of $\pi_{1}(M)$ in $\operatorname{Isom}(\widetilde{M})$. By the Theorem 7.2 of [Coo93], if $N(x, R)$ is the number of orbit points of $\Gamma \cdot x$ in $B(x, R)$, then there exists $C>0$ such that

$$
\frac{1}{C} e^{h R} \leq N(x, R) \leq C e^{h R}
$$

Hence if $F$ is a Dirichlet fundamental domain centered at $x$ for the action of $\Gamma$ and $D$ is the diameter of $F$, then

$$
\frac{1}{C} e^{-h D} \operatorname{Vol}(F) e^{h R} \leq \operatorname{Vol} B(x, R) \leq C e^{h D} \operatorname{Vol}(F) e^{h R} .
$$

The lemma now follows easily from the lower bound.

Lemma 6.2. Given an $\epsilon<\frac{\pi}{4}$ and any $R_{0}>0$, there is an sufficiently close enough to $h(g)$ such that if for all $R>R_{0}$ the $Q_{x}^{R}$ are $\epsilon$ close to a Möbius map $\mathcal{M}_{x}$, then $\bar{Q}_{x}$ is continuous and $2 \epsilon$ close to $\mathcal{M}_{x}$.

Proof. If all $Q_{x}^{R}$ are $\epsilon$ close to $\mathcal{M}_{x}$, then the values of $J_{x}(v)$ lie in the positive cone on a spherical ball of radius $\epsilon$ ball about $\mathcal{M}_{x}(v)$. With the bound on $\epsilon$ this 
cone is strictly convex. By choosing $s$ sufficiently close to $h(g)$ as in Lemma 6.1, the value of $\frac{\int_{0}^{R_{0}} e^{-s r} d v o l_{r}(v) d r}{\int_{0}^{\infty} e^{-s r} d v o l_{r}(v) d r}$ can be made as small as desired uniformly in $v$.

Hence for $s$ sufficiently close to $h(g), J_{x}$ is continuous since the $Q_{x}^{R}$ are continuous at each $v$ for all but a measure zero set of values of $R$. Moreover, after normalizing $J_{x}(v)$ to $\bar{Q}_{x}$, it will be at as close to $\epsilon$ distant from $\mathcal{M}_{x}$ as desired, so $2 \epsilon$ will do.

Proof of Theorem 1.3. By Lemma 6.2, under the hypotheses of the theorem, we may assume we have chosen $s$ small enough so that $\bar{Q}_{x}$ is $2 \epsilon$ close to a Möbius map which we shall denote $\mathcal{M}_{x}$.

Again we suppose that $x \in M$ is a critical point of $F_{s}$ and $u \in \operatorname{ker} d_{x} F_{s}$. As in the negatively curved case, we consider first the special case when $\bar{Q}_{x}=\mathcal{M}_{x}$ for all sufficiently large $R$. Adopting similar notation to the proof of 5.4, we have by assumption for all sufficiently large $R$, a common unique sphere $S_{t_{0}} \subset S_{x} M$ which is sent to an equator sphere by $\mathcal{M}_{x}$. Let $\left\{v_{+}, v_{-}\right\}$be the two (antipodal) unit vectors orthogonal to $\mathcal{M}_{x}\left(S_{t_{0}}\right)$ and let $H_{+}$and $H_{-}$be the hemispheres corresponding to $v_{+}$and $v_{-}$respectively. (Here again we assume we have chosen $v_{+}$such that $\mathcal{M}_{x}(u) \in H_{+}$.)

For the case $\bar{Q}_{x}=\mathcal{M}_{x}$, the conclusion again follows from the same proof as that of Lemma 5.4, using the measure $\tau_{x}^{s}$ that

$$
\int_{\mathcal{M}_{x}^{-1}\left(H_{+}\right)}\langle v, u\rangle\left\langle\mathcal{M}_{x}(v), v_{+}\right\rangle d \tau_{x}^{s}(v)>\int_{\mathcal{M}_{x}^{-1}\left(H_{-}\right)}\langle v, u\rangle\left\langle\mathcal{M}_{x}(v), v_{-}\right\rangle d \tau_{x}^{s}(v),
$$

contradicting Conditions (4) on $F_{s}$.

For the general case if $\bar{Q}_{x}$ is within $2 \epsilon$ of $\mathcal{M}_{x}$, then since the quantity

$$
\int_{\bar{Q}_{x}^{-1}\left(H_{+}\right)}\langle v, u\rangle\left\langle\bar{Q}_{x}(v), v_{+}\right\rangle d \tau_{x}^{s}(v)-\int_{\bar{Q}_{x}^{-1}\left(H_{-}\right)}\langle v, u\rangle\left\langle\bar{Q}_{x}(v), v_{-}\right\rangle d \tau_{x}^{s}(v),
$$

involves only bounded integrals and continuous maps whose limit as $\epsilon$ tends to 0 equals $\mathcal{M}_{x}$, it will become positive for $\epsilon$ sufficiently small, again contradicting Conditions (4).

Remark 6.3. As a final remark, we note that Theorem 1.3 adds to the long list of methods by which one can attempt to rule out the existence of homotopy spheres in dimension three. If $N$ is a closed hyperbolic 3-manifold, and $H$ were a homotopy sphere, then we could form any smooth connect sum to obtain $M=$ 
$H \# N$. There is a natural homotopy equivalence $f: M \rightarrow N$. If one could metrize a neighborhood of $H$ in the connect sum in such a way that the lifts of $H$ in the universal cover did not distort the asymptotic holonomy maps on $M$ too badly, then $f$ would be homotopic to a diffeomorphism by the main theorem. Milnor primality would then imply that $H$ was a sphere. However, achieving sufficient control this way on the $Q_{x}$ seems very difficult. For instance, in most dimensions greater than six a similar construction using exotic spheres produces non-diffeomorphic manifolds. In particular, the $Q_{x}$ could not be $\epsilon$-Möbius with the required $\epsilon$ in that case. Fortunately, Perelman's work makes any such effort unnecessary.

\section{REFERENCES}

[AS85] M. T. Anderson and R. Schoen, Positive harmonic functions on complete manifolds of negative curvature, Ann. of Math. (2) 121 (1985), no. 3, 429-461.

[BCG95] G. Besson, G. Courtois, and S. Gallot, Entropies et rigidités des espaces localement symétriques de courbure strictement négative, Geom. Funct. Anal. 5 (1995), no. 5, $731-799$.

[BCG96] G. Besson, G. Courtois, and S. Gallot, Minimal entropy and Mostow's rigidity theorems, Ergodic Theory Dynam. Systems 16 (1996), no. 4, 623-649.

[BCG98] G. Besson, G. Courtois, and S. Gallot, A real Schwarz lemma and some applications, Rend. Mat. Appl. (7) 18 (1998), no. 2, 381-410.

[BCG99] G. Besson, G. Courtois, and S. Gallot, Lemme de Schwarz réel et applications géométriques, Acta Math. 183 (1999), no. 2, 145-169.

[Bou95] M. Bourdon, Structure conforme au bord et flot géodésique d'un cat(-1)-espace, Enseign. Math. (2) 41 (1995), no. 1-2, 63-102.

[Con03] C. Connell, Minimal Lyapunov exponents, quasiconformal structures, and rigidity of non-positively curved manifolds, Ergodic Theory Dynam. Systems 23 (2003), no. 2, $429-446$.

[Con05] _ Smooth volume rigidity for manifolds with negatively curved targets, Available at http://arxiv.org/abs/0710.1104, 2005.

[Coo93] M. Coornaert, Mesures de Patterson-Sullivan sur le bord d'un espace hyperbolique au sens de Gromov, Pacific J. Math. 159 (1993), no. 2, 241-270.

[FJ89] F. T. Farrell and L. E. Jones, Negatively curved manifolds with exotic smooth structures, J. Amer. Math. Soc. 2 (1989), no. 4, 899-908.

[FJ93] _ Topological rigidity for compact non-positively curved manifolds, Differential geometry: Riemannian geometry (Los Angeles, CA, 1990), Proc. Sympos. Pure Math., vol. 54, Amer. Math. Soc., Providence, RI, 1993, pp. 229-274.

[FJ94a] Complex hyperbolic manifolds and exotic smooth structures, Invent. Math. 117 (1994), no. 1, 57-74. 
[FJ94b] _ Smooth rigidity and $C^{1}$-conjugacy at $\infty$, Comm. Anal. Geom. 2 (1994), no. 4, $563-578$

[FO04] F. T. Farrell and P. Ontaneda, Exotic structures and the limitations of certain analytic methods in geometry, Asian J. Math. 8 (2004), no. 4, 639-651.

[Gro87] M. Gromov, Hyperbolic groups, Essays in group theory, Math. Sci. Res. Inst. Publ., vol. 8, Springer, New York, 1987, pp. 75-263.

[GT87] M. Gromov and W. Thurston, Pinching constants for hyperbolic manifolds, Invent. Math. 89 (1987), no. 1, 1-12.

[Ham89] U. Hamenstädt, A new description of the Bowen-Margulis measure, Ergodic Theory Dynamical Systems 9 (1989), no. 3, 455-464.

[Man79] A. Manning, Topological entropy for geodesic flows, Ann. of Math. (2) 110 (1979), no. 3, 567-573.

[Mar70] G. A. Margulis, Certain measures that are connected with $\mathfrak{U}$-flows on compact manifolds, Funkcional. Anal. i Priložen. 4 (1970), no. 1, 62-76.

[Min07] I. Mineyev, Metric conformal structures and hyperbolic dimension, Conform. Geom. Dyn. 11 (2007), 137-163 (electronic).

[Pau96] F. Paulin, Un groupe hyperbolique est déterminé par son bord, J. London Math. Soc. (2) 54 (1996), no. 1, 50-74.

[Rob03] T. Roblin, Ergodicité et équidistribution en courbure négative, Mém. Soc. Math. Fr. (N.S.) (2003), no. 95, vi+96.

[Sam99] A. Sambusetti, Minimal entropy and simplicial volume, Manuscripta Math. 99 (1999), no. $4,541-560$.

[Sul84] D. Sullivan, Entropy, Hausdorff measures old and new, and limit sets of geometrically finite Kleinian groups, Acta Math. 153 (1984), no. 3-4, 259-277.

[Väi71] J. Väisälä, Lectures on n-dimensional quasiconformal mappings, Springer-Verlag, Berlin, 1971, Lecture Notes in Mathematics, Vol. 229.

[Yue91] C. B. Yue, Integral formulas for the Laplacian along the unstable foliation and applications to rigidity problems for manifolds of negative curvature, Ergodic Theory Dynam. Systems 11 (1991), no. 4, 803-819.

[Yue96a] _ Quasiconformality in the geodesic flow of negatively curved manifolds, Geom. Funct. Anal. 6 (1996), no. 4, 740-750.

[Yue96b] _ The ergodic theory of discrete isometry groups on manifolds of variable negative curvature, Trans. Amer. Math. Soc. 348 (1996), no. 12, 4965-5005.

\section{Chris Connell}

Email: connell@indiana.edu 